\title{
Significance of Membranous Urethral Length for Recovery From Postoperative Urinary Incontinence Following Holmium Laser Enucleation of the Prostate
}

\author{
Shintaro Oka, Keita Kobayashi, Kenji Matsuda, Kimio Takai \\ Department of Urology, Saiseikai Shimonoseki General Hospital, Yamaguchi, Japan
}

Purpose: The aim of this study was to determine the significance of the membranous urethral length (MUL), including the thickness of the urethral sphincter, for recovery from postoperative stress urinary incontinence (SUI) following holmium laser enucleation of the prostate (HoLEP).

Methods: We analyzed 78 patients who underwent HoLEP between June 2013 and September 2018, all of whom preoperatively received magnetic resonance imaging. MUL was measured using sagittal T2-weighted fast spin-echo images. The clini$\mathrm{cal}$ and anatomical factors associated with MUL were evaluated. The recovery time of urinary incontinence was compared between patients with a long MUL $(\geq 14 \mathrm{~mm})$ and a short MUL $(<14 \mathrm{~mm})$. SUI included both stress and mixed urinary incontinence. Continence was defined as complete dryness.

Results: The median MUL in patients without incontinence at 1 month postoperatively was significantly longer than the MUL in patients with incontinence $(15.3 \mathrm{~mm}$ vs. $12.7 \mathrm{~mm}, \mathrm{P}<0.001)$. The continence rates at 1 month after HoLEP in patients with longer MULs and shorter MULs were $80.4 \%$ and $30.0 \%$, respectively. The recovery time of urinary incontinence in patients with longer MULs $(\geq 14 \mathrm{~mm})$ was significantly shorter than that in patients with shorter MULs $(<14 \mathrm{~mm})(\log$-rank test, $\mathrm{P}=0.001)$. After 6 months, the continence rates in patients with longer MULs and shorter MULs were similar (97\%). MUL was significantly correlated with the recovery period of urinary incontinence $(r=-0.459, \mathrm{P}<0.001)$.

Conclusions: MUL was useful for predicting early recovery from urinary incontinence following HoLEP. This study provides evidence that postoperative urinary incontinence following a transurethral procedure for benign prostatic hyperplasia was associated with anatomical factors. A long MUL was associated with better tolerance to urinary sphincter damage by the transurethral procedure.

Keywords: Benign prostatic hyperplasia; Holmium lasers; Urinary incontinence; Urethral sphincters; Magnetic resonance imaging

- Research Ethics: This study was approved by the Institutional Review Board of Saiseikai Shimonoseki General Hospital (No. 1125).

- Conflict of Interest: No potential conflict of interest relevant to this article was reported.

\section{INTRODUCTION}

Holmium laser enucleation of the prostate (HoLEP) has emerged as an effective transurethral treatment option in patients with benign prostatic hyperplasia (BPH), and HoLEP enables complete prostate adenoma resection even in patients

Corresponding author: Shintaro Oka (iD https://orcid.org/0000-0001-7067-9678

Department of Urology, Saiseikai Shimonoseki General Hospital, 8-5-1

Yasuoka-cho, Shimonoseki, Yamaguchi 759-6603, Japan

E mail: s-oka@simo.saiseikai.or.jp

Submitted: January 29, 2020 / Accepted after revision: March 19, 2020 
with a large prostate volume. HoLEP reduces the Foley catheter placement time and enables a bloodless procedure [1]. However, a high frequency of postoperative stress urinary incontinence (SUI) following HoLEP is a serious issue. Urinary incontinence after HoLEP consists of stress and mixed urinary incontinence [2]. The incidence of SUI following transurethral resection of the prostate (TURP) and open prostatectomy has been reported as $8 \%[3]$ and $3 \%-9 \%[4,5]$, respectively. In contrast, the incidence of SUI following HoLEP has been reported to range from $10.7 \%$ to $38.4 \%$ [6-8]. A large prostate volume, long operation time, and performance of the procedure by surgeons with little experience with HoLEP have been reported as risk factors for postoperative incontinence [2,9]. Some of these risk factors are assumed to be caused by urinary sphincter injury. However, very few studies have investigated anatomical factors associated with urinary incontinence after surgery for $\mathrm{BPH}$. Although almost all cases of urinary incontinence following HoLEP improve within a year $[2,10]$, urinary incontinence often affects patients' quality of life. Therefore, estimating the recovery time of urinary incontinence is useful for surgeons who counsel patients about possible delays in continence following HoLEP.

It has been recognized that the membranous urethra plays an important role in urinary continence. The membranous urethral length (MUL) has been reported to be a predictive factor for continence recovery after radical prostatectomy. Patients with shorter MULs were found to be more likely to have incontinence following radical prostatectomy $[11,12]$. These reports show that the MUL is involved in the recovery of urethral sphincter damage causing urinary incontinence, and preoperative magnetic resonance imaging (MRI) is useful for visualizing the membranous urethra to measure MUL before radical prostatectomy. However, little information is available about the relationship between MUL and urinary incontinence following BPH surgery, including TURP and HoLEP.

The purpose of this study was to investigate the relationship between MUL measured by MRI and SUI following HoLEP. Damage to the urethral sphincter caused by transurethral procedures for BPH seems to be involved in postoperative incontinence. This study confirmed the significance of MUL in patients undergoing transurethral BPH surgery as a risk factor for postoperative urinary continence from an anatomical perspective.

\section{MATERIALS AND METHODS}

\section{Subjects}

We retrospectively reviewed the medical records of patients who underwent HoLEP. Patients who preoperatively received MRI were enrolled in the present study. MRI was performed for patients clinically suspected to have prostate cancer. When prostate cancer was suspected based on MRI, we performed prostate biopsy in all cases. Patients diagnosed with prostate cancer based on prostate biopsy findings were excluded from this study. Patients diagnosed with prostate cancer after HoLEP additionally received radical prostatectomy, radiation therapy, or active surveillance. Patients who underwent radical prostatectomy or radiation therapy after HoLEP, as well as patients with neurologic diseases that affected incontinence, were also excluded. Patients who had urinary incontinence before surgery were excluded, including those wearing pads for security without evidence of incontinence. A total of 78 patients who underwent HoLEP from June 2013 to September 2018 were identified. The patients' clinical information, including age, body mass index, history of diabetes, prostate volume, serum prostate-specific antigen (PSA) levels, history of acute urinary retention, operation time, enucleated prostate volume, and MUL measured by MRI, were collected. This study was approved by our Institutional Review Board of Saiseikai Shimonoseki General Hospital (No. 1125).

\section{Surgical Procedures and Postoperative Evaluation}

HoLEP operations were performed randomly by 4 surgeons at our institution. General or spinal anesthesia was administered in each case. All procedures were carried out using a 26F resectoscope (System-pro Laser Resectoscope, Olympus, Tokyo, Japan), a 100-W holmium: YAG laser (VersaPulse PowerSuite, Lumenis, Yokneam, Israel), and a 550- $\mu \mathrm{m}$ fiber (SlimLine 550, Lumenis, Yokneam, Israel). Our HoLEP technique was based on the anteroposterior dissection reported by Endo et al. [13]. Morcellation was performed using a tissue morcellator (VersaCut, Lumenis, Yokneam, Israel). After the procedure, a 22F Foley catheter was placed. The Foley catheter was removed on postoperative day 2. Continence was defined as complete dryness and no pad usage in the present study. Postoperative urinary incontinence included SUI, mixed urinary incontinence, and postvoid dribbling. Urinary incontinence was evaluated in a medical interview. All patients were asked about the presence of urinary incontinence at every medical examination after Ho- 
LEP. Follow-up was performed at 1, 3, 6, and 12 months postoperatively.

\section{MUL Measurement}

MRI was performed using a 1.5-T system (Ingenia CX, Philips Healthcare, Best, The Netherlands). A phased-array surface body coil was used. Midline sagittal T2-weighted turbo spinecho images were used to measure MUL, following previous reports $[14,15]$. MUL was measured as the distance from the

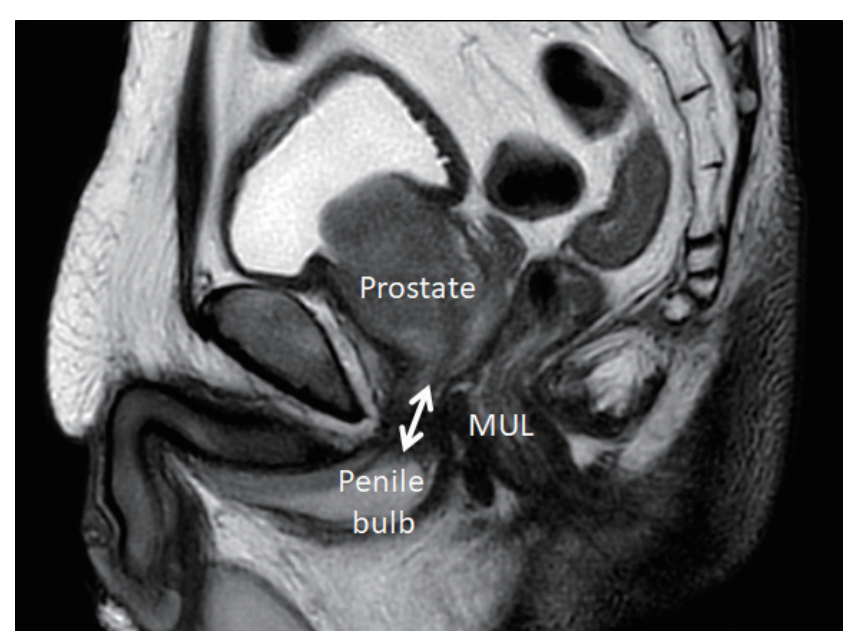

Fig. 1. T2-weighted sagittal magnetic resonance image for the measurement of membranous urethral length (MUL). The white arrow shows the MUL measured as the distance from the prostatic apex to the entry of the urethra into the penile bulb. prostatic apex to the entry of the urethra into the penile bulb (Fig. 1). Two urologists (SO and KK) independently evaluated several images and calculated the mean diameters.

\section{Statistical Analysis}

Statistical analyses were performed with SPSS ver. 12 (SPSS Inc., Chicago, IL, USA). Data are expressed as the means \pm standard deviation. The 2 groups were compared with the Mann-Whitney rank sum test. Logistic regression analysis was used to identify factors influencing the recovery of urinary incontinence. Kaplan-Meier analysis with log-rank testing was used to compare the recovery time of urinary incontinence. The Spearman rank-correlation test was used to examine the correlation between MUL and recovery time of urinary incontinence. A Pvalue $<0.05$ was considered to indicate statistical significance.

\section{RESULTS}

The continence rates at 1, 3, 6, and 12 months following HoLEP were $56 \%, 83 \%, 97 \%$, and $100 \%$, respectively. Patients were divided into 2 groups according to whether they had SUI at 1 month following HoLEP, and the clinical characteristics between the 2 groups were compared. Age and MUL were significantly different between the 2 groups (Table 1). The median patient ages were 68.5 and 74.4 years among patients without and with urinary incontinence, respectively, and patients without incontinence were significantly younger than patients with in-

Table 1. Comparison between patients with or without urinary incontinence at 1 month

\begin{tabular}{|c|c|c|c|}
\hline Variable & Patients without UI $(\mathrm{n}=44)$ & Patients with UI $(\mathrm{n}=34)$ & P-value $e^{a)}$ \\
\hline Age (yr) & $68.5 \pm 5.6$ & $74.4 \pm 8.2$ & 0.007 \\
\hline Body mass index $\left(\mathrm{kg} / \mathrm{m}^{2}\right)$ & $23.9 \pm 3.3$ & $23.5 \pm 3.2$ & 0.986 \\
\hline Diabetes & $6(13.6)$ & $2(5.8)$ & 0.246 \\
\hline Prostate volume $(\mathrm{mL})$ & $67.0 \pm 23.8$ & $68 \pm 22.6$ & 0.633 \\
\hline PSA level (ng/mL) & $5.8 \pm 5.1$ & $6.6 \pm 9.4$ & 0.260 \\
\hline MUL (mm) & $15.3 \pm 3.3$ & $12.7 \pm 2.8$ & $<0.001$ \\
\hline History of acute urinary retention & $8(18.2)$ & $11(32.3)$ & 0.163 \\
\hline Operative time (min) & $119 \pm 41.2$ & $142 \pm 39.3$ & 0.16 \\
\hline Enucleated prostate volume (g) & $34.5 \pm 20.3$ & $43.5 \pm 18.0$ & 0.205 \\
\hline Prostate cancer & $3(6.8)$ & $3(8.8)$ & 0.897 \\
\hline Anticholinergics & $7(15.9)$ & $4(11.7)$ & 0.601 \\
\hline
\end{tabular}

Values are presented as mean \pm standard deviations or number (\%)

UI, urinary incontinence; PSA, prostate-specific antigen; MUL, membranous urethral length.

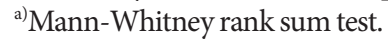


continence $(\mathrm{P}=0.007)$. The median MUL was $15.3 \mathrm{~mm}$ and 12.7 $\mathrm{mm}$ among patients without and with urinary incontinence, respectively. The MUL in patients without incontinence was significantly longer than that in patients with incontinence $(\mathrm{P}<0.001)$. At 3 months, the MUL in patients without incontinence was also significantly longer than that in patients with incontinence $(\mathrm{P}=0.001)$. In the multivariate analysis, age and MUL were significantly associated with early recovery of urinary continence (Table 2). Eleven patients took anticholinergic drugs because of continuing urgency after HoLEP. Other clinical characteristics or perioperative data were not significantly different between the 2 groups. Among the patients without SUI, 15.9\% (7 of 44) took anticholinergic drugs, and 11.7\% (4 of 34) of the patients with SUI took anticholinergic drugs. There was no significant difference in the incidence of urgency between the 2 groups.

Table 2. Multivariate logistic regression analysis of factors affecting recovery of urinary incontinence at 1 month after the operation

\begin{tabular}{lccc}
\hline Variable & Odds ratio & $95 \%$ CI & P-value \\
\hline Age & 1.090 & $1.010-1.180$ & 0.021 \\
MUL & 0.767 & $0.646-0.909$ & 0.002 \\
\hline
\end{tabular}

CI, confidence interval; MUL, membranous urethral length.

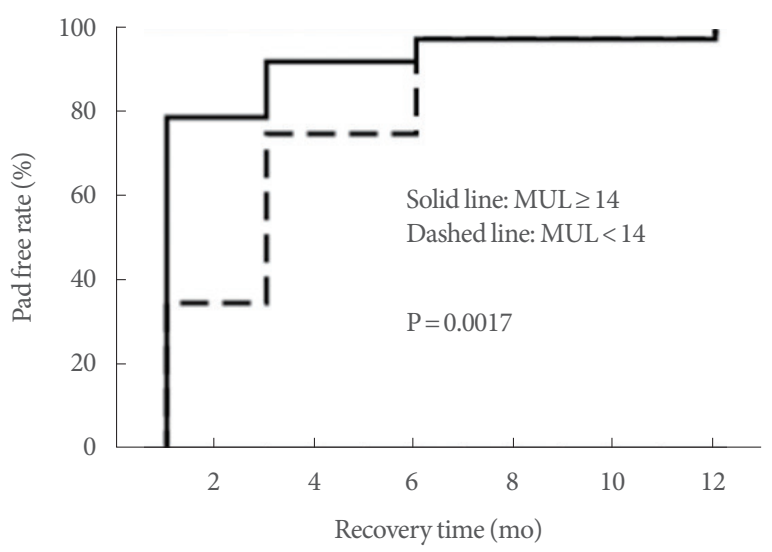

Fig. 2. A Kaplan-Meier curve shows the overall urinary continence rate after holmium laser enucleation of the prostate according to membranous urethral length (MUL). The patients with longer MULs ( $\geq 14 \mathrm{~mm}$ ) showed significantly earlier recovery than the patients with shorter MULs $(<14 \mathrm{~mm})$. The cutoff value of MUL was $14.0 \mathrm{~mm}$, which was the median value. There was no difference in the continence rate after 6 months between the 2 groups.
Among the 78 patients, the median MUL was $14.0 \mathrm{~mm}$ (range, 6.5 to $22.8 \mathrm{~mm}$ ). We divided the patients into 2 groups according to whether they had longer MULs $(\geq 14 \mathrm{~mm})$ or shorter MULs (<14 mm). At 1 month following HoLEP, the continence rates in patients with longer MULs and shorter MULs were $80.4 \%$ and $30.0 \%$, respectively. This difference in the continence rate between the 2 groups gradually disappeared. At 3 months, the continence rates in patients with longer MULs and shorter MULs were $92.6 \%$ and $72.9 \%$, respectively. At 6 months, the continence rate in the 2 groups was comparable at $97 \%$. Patients with longer MULs experienced significantly faster recovery of SUI compared with patients with shorter MULs (Fig. 2, log-rank test $\mathrm{P}=0.01$ ). These results indicate that MUL played a role in the early improvement of urinary continence following HoLEP.

We also investigated the correlation between MUL and the time required to achieve continence (Fig. 3). MUL was significantly correlated with the recovery time of postoperative incontinence $(\mathrm{r}=-0.459, \mathrm{P}<0.001)$. These results support the association of MUL with continence recovery.

\section{DISCUSSION}

Although the significance of MUL as a prognostic risk factor for overall continence recovery following radical prostatectomy has already been recognized, the significance of MUL in BPH surgery remains unclear. To our knowledge, this is the first re-

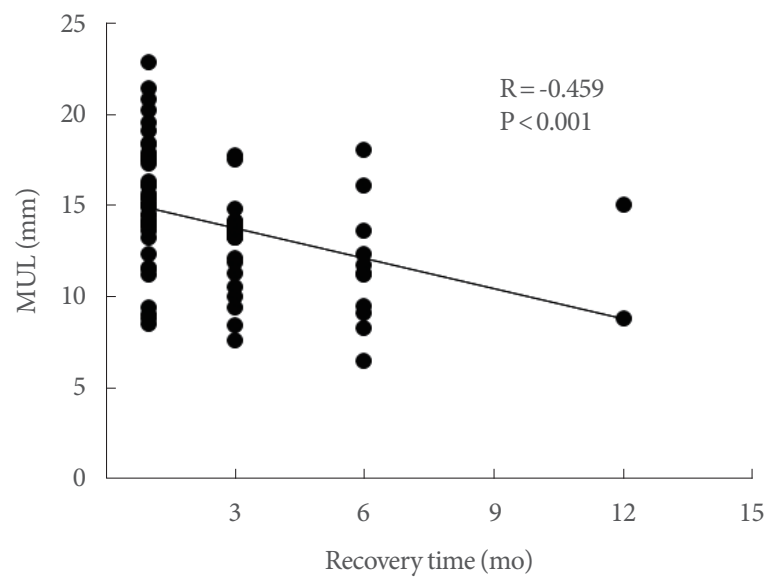

Fig. 3. Correlation of the timing of continence following holmium laser enucleation of the prostate (HoLEP) and membranous urethral length (MUL). MUL had a significant correlation with the time required to achieve urinary continence following HoLEP. 
port to evaluate the value of MUL as an anatomical factor in transurethral operations for $\mathrm{BPH}$. The present study indicates that MUL is an important anatomical factor affecting SUI associated with BPH surgery.

In the present study, $44 \%$ of patients had urinary incontinence at 1 month, and $17 \%$ of patients had urinary incontinence at 3 months. Compared with previously reported findings, the incontinence rate in the present study was higher than that observed with TURP or open prostatectomy. Patients using a pad for security due to rare incontinence or postvoid dribbling were included in this study, which may explain why the incontinence rate in our study was comparatively higher than that in other reports. In addition, the patients enrolled in this study had high PSA levels, so their age tended to be higher than the overall population of patients who undergo HoLEP. It was reported that $70 \%-90 \%$ of cases SUI following HoLEP improved spontaneously within 3 months, and almost all urinary incontinence resolved within 1 year $[2,10]$. In the present study, $98 \%$ of patients obtained complete dryness within 6 months. These results indicate that HoLEP is associated with a relatively high frequency of urinary incontinence compared with TURP; however, SUI following HoLEP is typically a transient phenomenon.

MUL was significantly associated with recovery of urinary incontinence following HoLEP (Table 2). In particular, there was a large difference in the incontinence rate in the first month (Fig. 3). Patients with longer MULs had a high continence rate (80.4\%) at 1 month; in contrast, patients with shorter MULs had a low continence rate at this time (30.0\%), indicating that MUL was associated with an early recovery of urinary incontinence after HoLEP. These results are congruent with the recovery of urinary incontinence following radical prostatectomy. A long MUL contributed to the recovery of urinary incontinence within 12 months after radical prostatectomy [12]. Urinary continence is mainly maintained by the continence zone in the posterior urethra. The urethral sphincter consists of 2 different muscles. The lissosphincter of smooth muscle in the inner layer and the rhabdosphincter of skeletal muscle in the outer layer wrapping the inner smooth muscle maintain urinary continence in cooperation $[16,17]$. A long MUL corresponds to a greater quantity of smooth muscle in the internal lissosphincter and skeletal muscle in the external rhabdosphincter. These anatomical factors seem to affect resistance to sphincter damage during HoLEP and are associated with a high resilience to this damage.
In the present study, the patients with an early recovery from urinary incontinence were younger than the patients with incontinence. In several studies, aging was also identified as an important risk factor for urinary incontinence following BPH surgery $[2,18,19]$. It is suspected that younger patients have thicker and stronger urethral sphincters than older patients. We investigated the correlation between patient age and MUL; however, no significant association was found between these factors in the present study. Detectable differences in MUL using MRI between younger patients and older patients were not identified in the present study; however, the urethral sphincter in older patients may be more vulnerable to damage than those in younger patients.

HoLEP is considered to be more difficult than other transurethral procedures for $\mathrm{BPH}$, with a steeper learning curve [20]. The HoLEP surgical technique during the learning period is an important risk factor for postoperative urinary incontinence $[9,21]$. Unintended resection of the urinary sphincter or excessive stretching of the internal lissosphincter of the smooth muscle around the apical gland may cause urinary incontinence after HoLEP. We performed anteroposterior dissection HoLEP, as reported by Endo et al. [13], to prevent damage to the internal lissosphincter of the smooth muscle. In this procedure, the lateral adenoma is dissected from the 12-oclock to the 6-oclock position, imitating the peeling procedure of open prostatectomy. This procedure separates the sphincter from the urethra, preserving the internal muscle of the sphincter without excessive stretching of the sphincter. Despite improvements in the surgical technique of HoLEP, the incidence of urinary incontinence following HoLEP is still a critical problem, especially for novice surgeons. We believe that the preoperative measurement of MUL is especially useful for novice surgeons to prepare for the operation. If a patient is found to have a short MUL when evaluated before surgery, particular attention is needed to preserve the urethral sphincter around the apex of the prostate.

A long operation time was reported as a risk factor for urinary incontinence following HoLEP [2]. Because HoLEP requires a wider range of scope motion than TURP, the urethral sphincters are exposed to more force and sustain more damage by resectoscope sheath manipulation during the HoLEP procedure. The increasing damage with longer operation times may be associated with urinary incontinence. The operation time tends to be relatively long in patients with a large prostate volume, a history of urinary tract infection, or urinary retention [22]. Surgeons need to perform the HoLEP procedure particu- 
larly carefully to avoid applying excessive pressure or traction on the urinary sphincter, especially in patients with shorter MULs.

The present study has several limitations. First, the HoLEP procedures were performed by 4 surgeons, so differences in technique and experiences may have produced variability in the outcomes. However, we analyzed the difference in the continence rate among the surgeons, and there was no significant difference. We therefore think that differences in surgical technique among the surgeons did not influence the main results of this study. Second, the small sample size is also a limitation. MRI is not necessarily performed before BPH surgery unless prostate cancer is suspected before HoLEP. Therefore, patients with MRI information constitute a subset of the total group of patients who received HoLEP. In the present study, a significant correlation was identified between MUL and the recovery time of urinary incontinence (Fig. 3), suggesting that individual variation was limited. Therefore, we believe that the sample size was sufficient in this study. Third, this study had selection bias for patients. The patients in this study had higher PSA levels than general patients, suggesting that they had prostatic inflammation or prostate cancer. In fact, we identified patients with prostatic inflammation in this study. We did not investigate urodynamics in the present study. In the future, further studies including urodynamic investigations are needed to clarify the involvement of urinary sphincter function in continence outcomes.

MUL is a significant factor predicting early recovery from SUI following HoLEP. Anatomical factors are also important for postoperative urinary incontinence. This study indicates that a longer MUL has a positive effect on early recovery from urinary incontinence caused by sphincter damage during procedures such as radical prostatectomy. Preoperative information on MUL is helpful during transurethral procedures for $\mathrm{BPH}$ to decrease postoperative urinary incontinence. This finding is important because postoperative urinary incontinence remains a major concern for surgeons and patients.

\section{REFERENCES}

\section{Montorsi F, Naspro R, Salonia A, Suardi N, Briganti A, Zanoni M,} et al. Holmium laser enucleation versus transurethral resection of the prostate: results from a 2-center, prospective, randomized trial in patients with obstructive benign prostatic hyperplasia. J Urol 2004;172:1926-9.
2. Nam JK, Kim HW, Lee DH, Han JY, Lee JZ, Park SW. Risk factors for transient urinary incontinence after holmium laser enucleation of the prostate. World J Mens Health 2015;33:88-94.

3. Hogewoning CRC, Meij LAM, Pelger RCM, Putter H, Krouwel EM, Elzevier HW. Sling surgery for the treatment of urinary incontinence after transurethral resection of the prostate: New data on the virtue male sling and an evaluation of literature. Urology 2017; 100:187-92.

4. Serretta V, Morgia G, Fondacaro L, Curto G, Lo bianco A, Pirritano $\mathrm{D}$, et al. Open prostatectomy for benign prostatic enlargement in southern Europe in the late 1990s: a contemporary series of 1800 interventions. Urology 2002;60:623-7.

5. Tubaro A, Carter S, Hind A, Vicentini C, Miano L. A prospective study of the safety and efficacy of suprapubic transvesical prostatectomy in patients with benign prostatic hyperplasia. J Urol 2001; 116:172-6.

6. Krambeck AE, Handa SE, Lingeman JE. Experience with more than 1,000 holmium laser prostate enucleations for benign prostatic hyperplasia. J Urol 2010;183:1105-9.

7. Cho KJ, Koh JS, Choi JB, Kim JC. Factors associated with early recovery of stress urinary incontinence following holmium laser enucleation of the prostate in patients with benign prostatic enlargement. Int Neurourol J 2018;22:200-5.

8. Shah HN, Mahajan AP, Hegde SS, Bansal MB. Peri-operative complications of holmium laser enucleation of the prostate : experience in the first 280 patients, and a review of literature. BJU int 2007; 100:94-101.

9. Lerner LB, Tyson MD, Mendoza PJ. Stress incontinence during the learning curve of holmium laser enucleation of the prostate. J Endourol 2010;24:1655-8.

10. Vavassori I, Valenti S, Naspro R, Vismara A, Dell'Acqua V, Manzetti $\mathrm{A}$, et al. Three-year outcome following holmium laser enucleation of the prostate combined with mechanical morcellation in 330 consecutive patients. Eur Urol 2008;53:599-606.

11. Coakley FV, Eberhardt S, Kattan MW, Wei DC, Scardino PT, Hricak H. Urinary continence after radical retropubic prostatectomy: Relationship with membranous urethral length on preoperative endorectal magnetic resonance. J Urol 2002;168:1032-5.

12. Mungovan SF, Sandhu JS, Akin O, Smart NA, Graham PL, Patel MI. Preoperative membranous urethral length measurement and continence recovery following radical prostatectomy: A systematic review and meta-analysis. Eur Urol 2017;71:368-78.

13. Endo F, Shiga Y, Minagawa S, Iwabuchi T, Fujisaki A, Yashi M, et al. Anteroposterior dissection HoLEP: A modification to prevent transient stress urinary incontinence. Urology 2010;76:1451-5. 
14. Tienza A, Hevia M, Benito A, Pascual JI, Zudaire JJ, Robles JE. MRI factors to predict urinary incontinence after retropubic/laparoscopic radical prostatectomy. Int Urol Nephrol 2015;47:1349-9.

15. Kitamura K, China T, Kanayama M, Nagata M, Isotani S, Wakuoto Y, et al. Significant association between urethral length measured by magnetic resonance imaging and urinary continence recovery after robot-assisted radical prostatectomy. Prostate Int 2019;7:54-9.

16. Burnett AL, Mostwin JL. In situ anatomical study of the male urethral sphincteric complex: Relevance to continence preservation following major pelvic surgery. J Urol 1998;160:1301-6.

17. Walz J, Burnett AL, Costello AJ, Eastham HA, Graefen M, Guillonneau $B$, et al. A critical analysis of the current knowledge of surgical anatomy related to optimization of cancer control and preservation of continence and erection in candidates for radical prostatectomy. Eur Urol 2010;57:179-92.

18. Xu N, Chen S, Xue X, Wei Y, Zheng Q, Li X, al. Older age and larger prostate volume are associated with stress urinary incontinence after plasmakinetic enucleation of the prostate. Biomed Res Int 2017:6923290.

19. Bruschini H, Simonetti R, Antunes AA, Srougi M. Urinary incontinence following surgery for BPH: The role of aging on the incidence of bladder dysfunction. Int Braz J Urol 2011;37:380-6.

20. Robert G, Cornu JN, Fourmarier M, Saussine C, Descazeaud A, Azzouzi AR, et al. Multicentre prospective evaluation of the learning curve of holmium laser enucleation of the prostate (HoLEP). BJU Int 2016;117:495-9.

21. Kobayashi S, Yano M, Nakayama T, Kitahara S. Predictive risk factors of postoperative urinary incontinence following holmium laser enucleation of the prostate during the initial learning period. Int Braz J Urol 2016;42:740-6.

22. Monn MF, El Tayeb M, Bhojani N, Mellon MJ, Sloan JC, Boris RS, et al. Predictors of enucleation and morcellation time during holmium laser enucleation of the prostate. Urology 2015;86:338-42. 